\title{
Anomalous right coronary artery originates from the mid of left anterior descending coronary artery and coursing between aorta and pulmonary artery
}

\author{
Xuguang Qin ${ }^{1 *}$, Weiguo Xiong ${ }^{2}$ and Chunpeng $\mathbf{L u}^{2}$ \\ ${ }^{1}$ Department of cardiology, Beijing North Asia Orthopedics Hospital, China \\ ${ }^{2}$ Department of cardiology, First Affiliated Hospital of Tsinghua University, China
}

\section{Case Presentation}

Coronary artery anomalies (CAAs) are present at birth but are usually asymptomatic and are found during coronary angiography or multi-slice computed tomography (MSCT) detection. Their prevalence is less than $1.3 \%$ based published series [1-4]. The most common coronary anomaly is separate origination of the left anterior descending coronary artery (LAD) and left circumflex coronary artery (LCX) from left sinus of Valsalva. The second most common anomaly is origination of the LCX artery from the right coronary artery (RCA) or right sinus of Valsalva. Herein, we present a case is that the anomalous RCA a part arises from mid of left anterior descending coronary artery and courses between aorta and pulmonary artery, the other originates from the distal of left circumflex coronary artery. It is belonging to single coronary artery (SCA). The case is rare. we bring forth the case in an attempt to highlight its significance, and make cardiologist understood what important the anomalies are.

A 42-year-old man presented with a history of progressive angina pectoris of 3 months duration. He had a ten-year history of diabetes mellitus and hypertension. Electrocardiography and echocardiography examination is normal. Coronary angiogram was performed through trans-radial access. An angiogram demonstrated a single coronary artery: the anomalous right coronary artery (RCA) partly originated from the mid of left anterior descending coronary artery (LAD) and the other part arose from the distal of left circumflex artery (LCX). There was $80-90 \%$ stenosis in the proximal of the anomalous RCA segment, and the left anterior descending coronary artery (LAD), LCX were patent (Figures 1-3). A 6Fr left EBU 3.5 guiding catheter was engaged to the left coronary system to perform percutaneous coronary intervention (PCI) on the anomalous RCA and one stent was deployed. The final angiographic result was excellent.

Befoe the coronary angiography, a 64-slice computed tomography (MSCT) of the heart was performed on a 64-slice machine (Philips 64 Slice, Philips, Netherland). The results showed the anomalous right coronary artery partly originating from the mid of the left anterior descending coronary artery, and the other part arising from the distal of left circumflex coronary artery (Figures 4-6). There was severe stenosis in the proximal of the anomalous right coronary artery. The anomalous RCA was classified as single coronary anomaly, L-IIb subtype.

\section{Discussion}

The most common an anomalous right coronary artery (RCA) is that originates from the aortic trunk of ascending artery or left sinus

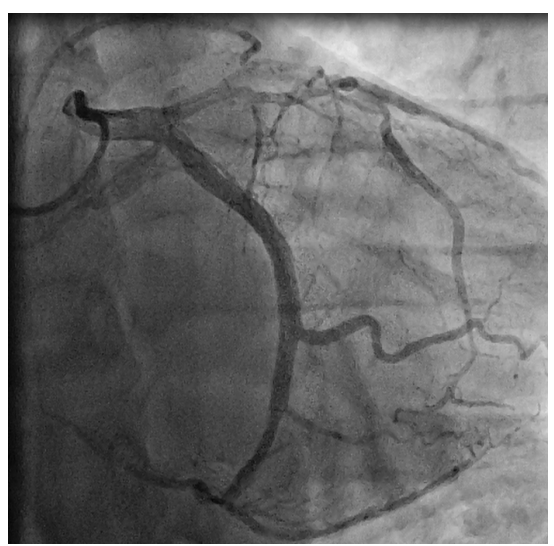

Figure 1. AP caudal view demonstrates the anomalous right coronary artery originating from the mid of left anterior descending coronary artery

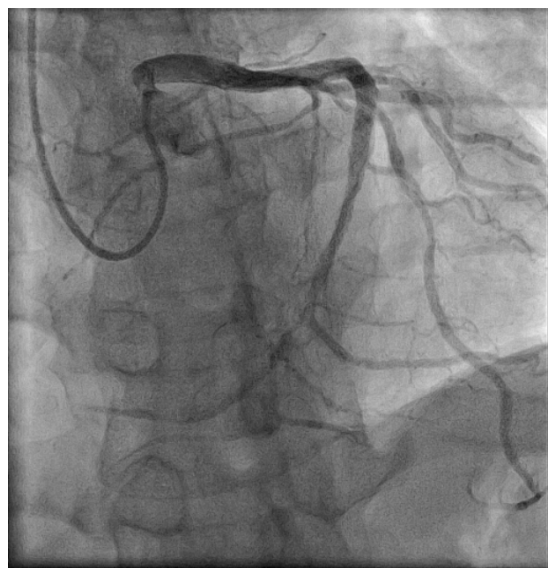

Figure 2. RAO cranial view demonstrates the other part of anomalous right coronary artery originating from the distal of left circumflex coronary artery

${ }^{*}$ Correspondence to: Xuguang Qin, Department of Cardiology, Beijing North Asia Orthopedics Hospital, Fangshan 102445, China, E-mail: qin xuguang0712@163.com

Key words: Coronary anomaly, single coronary anomaly, anomalous right coronary artery, multi-slice computed tomography

Received: May 10, 2020; Accepted: May 14, 2020; Published: May 22, 2020 


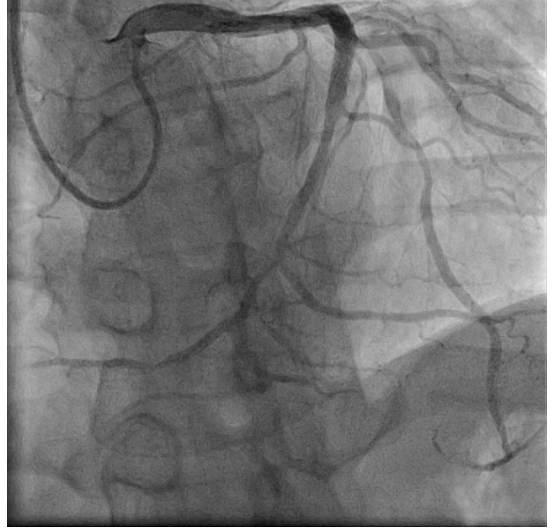

Figure 3. RAO cranial view demonstrates there is $70 \%$ stenosis in mid of left anterior descending coronary coronary artery segment

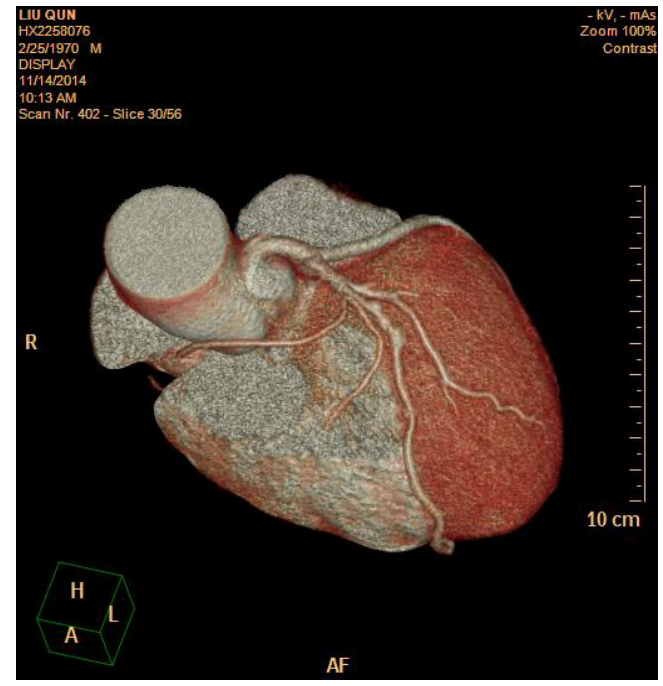

Figure 4. Reconstructed three-dimensional image obtained by volume-rendering technique demonstrates part of the anomalous RCA course between aorta and pulmonary artery

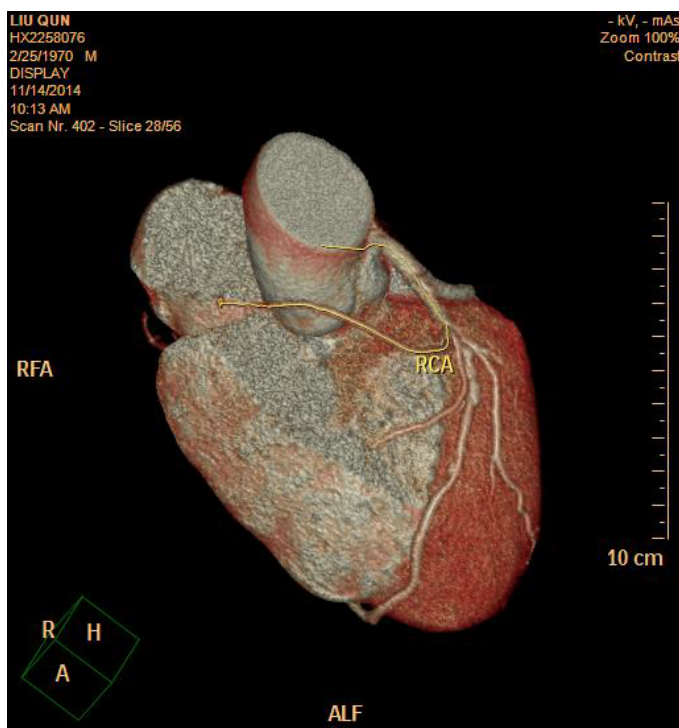

Figure 5. Reconstructed three-dimensional image obtained by volume-rendering technique demonstrates part of the anomalous RCA originating from the mid of the left anterior descending coronary artery

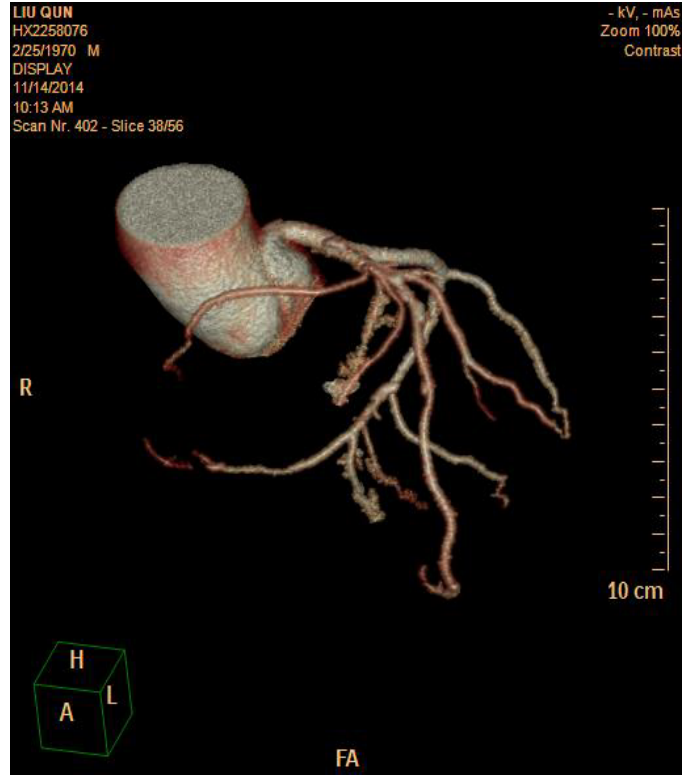

Figure 6. Reconstructed three-dimensional image obtained by volume-rendering technique demonstrates the other part of the anomalous RCA originating from the distal of the left circumflex coronary artery

of Valsalva. The case we presented is the anomalous RCA arises from the mid of left anterior descending coronary artery. It is belonging to single coronary artery (SCA) [1]. The anomalous coronary artery is first designated with "R" or "L" depending upon whether the ostium is located in the right or left sinus of Valsalva. It is then designated as group I, II, III. Group I have anatomical course of either a right or left coronary artery. Group II anomalies arise from the proximal part of the normal right or left coronary artery and cross the base of the heart before assuming the normal position of the inherent coronary artery. Group III describes the anomaly where the LAD and LCX arise separately from the proximal part of the normal right coronary artery. The L-I pattern occurs where the right coronary artery is congenitally absent and the LCX is markedly dominant (Figures 1-6). The LCX artery provides the posterior descending branch and ascends in the right $\mathrm{AV}$ groove where it provides branches to the right atrium and right ventricle. The extremely rare Group I anomalies generally have a benign clinical course [2]. But our case also have malignant course. PCI was performed to the anomalous right coronary artery and one stent was deployed into it through trans-radial approach. The patient has nonischemic symptoms on physical exertion during 6-year follow-up.

Coronary anomalies are usually detected during coronary angiography. However, X-ray angiography is limited by its inability to provide information regarding the spatial orientation of the anomalous artery with regarding to the surrounding cardiovascular structures $[3,4]$. MSCT technique has the potential to accurately visualize the coronary artery [5] and clearly demonstrate the surrounding cardiovascular structures of the anomalous coronary artery [68]. In the present case, the MSCT images clearly demonstrated the anomalous right coronary artery originating from the mid of left anterior descending coronary artery and coursing between aorta and pulmonary artery. So, we bring forth the extremely case in an attempt to highlight their significance, and make cardiologist understood what important the anomalies are. 


\section{References}

1. Lipton MJ, Barry WH, Obrez I, Silverman JF, Wexler L (1979) Isolated single coronary artery: diagnosis, angiographic classification, and clinical significance. Radiology 130 : 39-47. [Crossref]

2. Yamanaka O, Hobbs RE (1990) Coronary artery anomalies in 126,595 patients undergoing coronary arteriography. Cathet Cardiovasc Diag 21: 28-40. [Crossref]

3. Sheth M, Dovnarsky M, Cha SD, Kini P, Maranhao V (1998) Single coronary artery: right coronary artery originating from distal left circumflex. Cathet Cardiovasc Diagn 14: 180-181. [Crossref]

4. Roberts WC, Siegel RJ, Zipes DP (1982) Origin of the right coronary artery from the left sinus of Valsalva and its functional consequences: Analysis of 10 necropsy patients. Am J Cardiol 49: 863-868. [Crossref]
5. Barth III CW, B ray M, Roberts WC (1986) Sudden death in infancy associated with origin of both left main and right coronary arteries from a common ostium above the left sinus of Valsalva. Am J Cardiol 57: 365-366. [Crossref]

6. Angelini P, Velasco JA, Flamm S (2002) Coronary anomalies. Incidence, pathophysiology, and clinical elevance. Circulation 105: 2449-2454. [Crossref]

7. Dongyong He, Weiguo Xiong, Xuguang Qin, Chunpeng Lu, Hongliang Li, etal. (2014) Coronary artery anomalies: the left main coronary artery or left anterior descending coronary artery originating from the proximal of right coronary artery. Chin Med J 127: 2392-2394. [Crossref]

8. Tariq S, Muddassir S, Patel JK (2012) Single coronary artery anomaly: Branching of left coronary artery from right coronary artery with 2 distinct patterns. $J$ Invasive Cardiol 24: E67-71. [Crossref]

Copyright: (C2020 Qin X. This is an open-access article distributed under the terms of the Creative Commons Attribution License, which permits unrestricted use, distribution, and reproduction in any medium, provided the original author and source are credited. 